\title{
The effect of pmpR on the type III secretion system in Pseudomonas aeruginosa
}

\author{
LIANG HaiHua $^{\dagger}$, KONG WeiNa $^{\dagger}$, SHEN Tuo, DUAN JiaLi \& DUAN KangMin* \\ Key Laboratory of Resources Biology and Biotechnology in Western China, Ministry of Education, Faculty of Life Sciences, Northwest University, \\ Xi'an, Shaanxi 710069, China
}

Received June 21, 2011; accepted November 15, 2011; published online March 8, 2012

\begin{abstract}
The type III secretion system (T3SS) plays important roles in Pseudomonas aeruginosa pathogenicity. Previously, we reported that the uncharacterized protein PmpR could regulate $p q s R$, an important regulator in the quorum-sensing system, by directly binding to its promoter region. As the T3SS is controlled by the quorum-sensing system, here, we investigated the relationship between PmpR and the T3SS. Our data showed that expression of the T3SS genes exoS, exoY, exoT, and exsD was dramatically increased in a $p m p R$-deletion mutant compared with that in the wild-type $P$. aeruginosa strain PAO1. Data from DNA mobility assays indicated that PmpR affects the T3SS indirectly. It is unlikely that PmpR controls the T3SS via the Pseudomonas quinolone signal (PQS) because the PQS negatively regulates the T3SS, while $p m p R$ negatively regulates the PQS. The effect of PmpR on the T3SS seems to be independent of the PQS; further investigation is required to uncover the underlying regulatory pathways.
\end{abstract}

\section{P. aeruginosa, pmpR, PQS, type III secretion system}

Citation: $\quad$ Liang H H, Kong W N, Shen T, et al. The effect of pmpR on the type III secretion system in Pseudomonas aeruginosa. Chin Sci Bull, 2012, 57: 2413-2418, doi: 10.1007/s11434-011-4941-x

Pseudomonas aeruginosa is an opportunist pathogen that causes serious infectious diseases in humans. Its infection in the lungs of cystic fibrosis patients is the main cause of morbidity and mortality in these patients. Complex factors are involved in $P$. aeruginosa pathogenicity, and among them, quorum-sensing (QS) and the Type III secretion system (T3SS) play important roles.

$P$. aeruginosa possesses at least 2 well-defined acylhomoserine lactone (HSL)-based quorum-sensing systems, las and $r h l$, which control numerous virulence factor genes including las $A$, las $B$, and $r h l A[1,2]$. Each system comprises a transcriptional activator and an autoinducer synthase. The las system consists of the transcriptional activator LasR, and the QS signal synthase LasI that directs the biosynthesis of N-3-oxo-dodecanoyl-HSL ( $3 \mathrm{OC}_{12}$-HSL). The $r h l$ system consists of the transcriptional activator RhlR, and the synthase $\mathrm{Rhl}$ that is responsible for the biosynthesis of N-butanoyl-HSL ( $\mathrm{C}_{4}$-HSL). 3O- $\mathrm{C}_{12}$-HSL and $\mathrm{C}_{4}$-HSL acti-

$\uparrow$ These authors contributed equally to this work.

*Corresponding author (email: kduan@nwu.edu.cn) vate the transcription of LasR and RhlR respectively [3]. Activation of RhlR by $\mathrm{C}_{4}$-HSL regulates the production of rhamnolipid, hydrogen cyanide, pyocyanin, and proteins of the T3SS pathway. Recent microarray analyses have revealed that the las and $r h l$ systems also regulate the expression of many additional $P$. aeruginosa genes $[4,5]$.

Besides $3 \mathrm{O}-\mathrm{C}_{12}$-HSL and $\mathrm{C}_{4}-\mathrm{HSL}, P$. aeruginosa produces additional intercellular signaling molecules termed the AHQ signals [6]. The major AHQ signals include 2-heptyl-3-hydroxy-4-quinolone (the Pseudomonas quinolone signal, PQS) and 2-heptyl-4-quinolone [6-8]. The PQS is synthesized by enzymes encoded by the pqsABCDE and $p h n A B$ operons as well as $p q s H$ [8]. The transcription of these two operons is regulated by the PQS transcriptional regulator PqsR, also known as MvfR [8]. Mutations in pqsR interfere with PQS synthesis and affect the production of pyocyanin and other QS-controlled virulence factors [8,9].

The T3SS is a highly specialized protein secretion apparatus that facilitates the translocation of effector proteins from the bacterial cytoplasm directly into host cells. The 
T3SS plays important roles in $P$. aeruginosa pathogenesis especially during acute infections. In $P$. aeruginosa, a single set of genes encodes the structural components of the T3SS. This pathogen has 4 known effectors, ExoS, ExoT, ExoU, and ExoY [10]. These T3SS effectors are major virulence factors of $P$. aeruginosa. All T3SS genes, both structural genes and effectors, such as exoS, are under the direct transcriptional control of ExsA [11]. Moreover, ExsA interacts with and is influenced by ExsC, ExsD, and ExsE. These protein interactions, which are affected by $\mathrm{Ca}^{2+}$ concentration and T3SS secretory activity, eventually determine the availability of free ExsA and hence T3SS activity $[12,13]$. Intriguingly, the T3SS is also affected by the QS system in $P$. aeruginosa. It has been reported that the T3SS is negatively regulated by the $r h l$ system [14].

In our previous study, we found that PmpR could directly bind to the $p q s R$ promoter region and regulate the PQS. Considering that the PQS is connected to the $r h l$ system, in this study we investigated the relationships between PmpR and the T3SS. Our data indicate that the PQS system negatively controls the T3SS and that PmpR indirectly affects the T3SS, possibly via a pathway independent of the PQS.

\section{Materials and methods}

\subsection{Bacterial strains and media}

The bacterial strains and plasmids used in this study are listed in Table 1. P. aeruginosa wild-type strain and derivatives were routinely grown at $37^{\circ} \mathrm{C}$ on $\mathrm{LB}$ agar plates or in LB broth with orbital shaking at $200 \mathrm{r} / \mathrm{min}$. Antibiotics were purchased from Sigma (St. Louis, USA) and used at the following concentrations where appropriate: for $E$. coli in all media, kanamycin at $50 \mu \mathrm{g} \mathrm{mL} \mathrm{m}^{-1}$, tetracycline at 15 $\mu \mathrm{g} \mathrm{mL} \mathrm{L}^{-1}$, and ampicillin at $100 \mu \mathrm{g} \mathrm{mL}^{-1}$; for $P$. aeruginosa, gentamicin at $75 \mu \mathrm{g} \mathrm{mL}^{-1}$ in $\mathrm{LB}$ or $150 \mu \mathrm{g} \mathrm{mL}^{-1}$ in Pseudomonas Isolation Agar (PIA), tetracycline at $75 \mu \mathrm{g} \mathrm{mL}^{-1}$ in $\mathrm{LB}$ or $200 \mu \mathrm{g} \mathrm{mL}^{-1}$ in PIA, carbenicillin at $500 \mu \mathrm{g} \mathrm{mL}^{-1}$ in PIA, trimethoprim at $300 \mu \mathrm{g} \mathrm{mL}^{-1}$ in all media.

\subsection{Construction of the promoter-reporter fusion plasmids}

The plasmid pMS402 carrying a promoter-less luxCDABE reporter gene cluster was used to construct promoterluxCDABE reporter fusions of exs $D$, as reported previously $[15,20]$. The promoter regions of exs $D$ were PCR-amplified using high-fidelity $P f x$ DNA polymerase and primers synthesized according to the $P$. aeruginosa PAO1 strain genome data [21]. The promoter regions were cloned into the Xho I-BamH I site upstream of the lux genes in pMS402. Then, the resultant plasmid was digested with Pac I, and the larger fragment was isolated by gel extraction. Next, the purified DNA was ligated with the CTX6.1 plasmid, which was also digested with Pac I. The construct was integrated into the PAO1 chromosome by bi-parental mating [17].

Table 1 Strains and plasmids used in this studya)

\begin{tabular}{|c|c|c|}
\hline Strains or plasmids & Relevant feature & Source or reference \\
\hline \multicolumn{3}{|l|}{ P. aeruginosa } \\
\hline PAO1 & $P$. aeruginosa wild type & This laboratory \\
\hline$\Delta p m p R$ & $p m p R$ replacement mutant of $\mathrm{PAO} 1, \mathrm{Gm}^{\mathrm{r}}$ & [15] \\
\hline$\Delta p q s R$ & $p q s R$ replacement mutant of $\mathrm{PAO} 1, \mathrm{Gm}^{\mathrm{r}}$ & This study \\
\hline \multicolumn{3}{|l|}{ E. coli } \\
\hline $\mathrm{DH} 5 \alpha$ &  & Invitrogen \\
\hline SM10 & Mobilizing strain, RP4 integrated in the chromosome; $\mathrm{Kn}^{\mathrm{r}}$ & [16] \\
\hline \multicolumn{3}{|l|}{ Plasmids } \\
\hline pEX18Ap & ${ }^{o r i} T^{+} s a c B^{+}$gene replacement vector with multiple-cloning site from $\mathrm{pUC} 18, \mathrm{Ap}^{\mathrm{r}}$ & {$[17]$} \\
\hline pZ1918Gm & Source plasmid of $\mathrm{Gm}^{\mathrm{r}}$ cassette & [18] \\
\hline pRK2013 & Broad-host-range helper vector; $\mathrm{Tra}^{+}, \mathrm{Kn}^{\mathrm{r}}$ & [19] \\
\hline CTX6.1 & Integration plasmid; Tet $^{\mathrm{r}}$ & This laboratory \\
\hline $\mathrm{HTb}-p m p R$ & Protein expression construct, $p m p R$ cloned in $\mathrm{HTb}$ vector & {$[15]$} \\
\hline pKD-exs $D$ & pMS402 containing exs $D$ promoter region; $\mathrm{Kn}^{\mathrm{r}}$ & This study \\
\hline pKD-exos & pMS402 containing exoS promoter region; $\mathrm{Kn}^{\mathrm{r}}$ & {$[20]$} \\
\hline pKD-exoY & pMS402 containing exoY promoter region; $\mathrm{Kn}^{\mathrm{r}}$ & [20] \\
\hline pKD-exoT & pMS402 containing exoT promoter region; $\mathrm{Kn}^{\mathrm{r}}$ & {$[20]$} \\
\hline CTX-exs D & Integration plasmid, CTX6.1 with a fragment of $\mathrm{pKD}-\operatorname{exs} D ; \mathrm{Kn}^{\mathrm{r}}, \mathrm{Tmp}^{\mathrm{r}}, \mathrm{Tc}^{\mathrm{r}}$ & This study \\
\hline CTX-exos & Integration plasmid, CTX6.1 with a fragment of $\mathrm{pKD}-\operatorname{exo} S ; \mathrm{Kn}^{\mathrm{r}}, \mathrm{Tmp}^{\mathrm{r}}, \mathrm{Tc}^{\mathrm{r}}$ & This study \\
\hline CTX-exoY & Integration plasmid, CTX6.1 with a fragment of $\mathrm{pKD}-\operatorname{exo} Y ; \mathrm{kn}^{\mathrm{r}}, \mathrm{Tmp}^{\mathrm{r}}, \mathrm{Tc}^{\mathrm{r}}$ & This study \\
\hline CTX-exoT & Integration plasmid, CTX6.1 with a fragment of pKD-exoT; $\mathrm{kn}^{\mathrm{r}}, \mathrm{Tmp}^{\mathrm{r}}, \mathrm{Tc}^{\mathrm{r}}$ & This study \\
\hline
\end{tabular}

a) Kan, kanamycin; Tc, tetracycline; Ap, ampicillin; Gm, gentamicin; Tc, tetracycline; Cb, carbenicillin; Tmp, trimethoprim. 
Chromosome-integrated reporters for exoS, exoY, and exoT were constructed in the same way by using previously reported fusion constructs (Table 1). DNA manipulation, PCR, and transformation were performed following standard procedures. Cloned promoter sequences were confirmed by DNA sequencing.

\subsection{Construction of a pqsR-deletion mutant}

For gene replacement, the previously described $s a c B$-based strategy was employed [17]. A suicide plasmid of pKDpqsR for gene replacement was constructed by inserting the BamH I-digested $\mathrm{Gm}^{\mathrm{r}}$-lacZ cassette derived from pZ1918lacZ [18] in between 2 PCR fragments of $p q s R$ cloned into pEX18Ap. The upstream fragment of $p q s R$ was amplified using the primers L1 and H1 (Table 2). The downstream region of $p q s R$ was generated using primers $\mathrm{L} 2$ and $\mathrm{H} 2$. The pKDpqs $R$ plasmid was transferred to PAO1 using a tri-parental mating procedure employing the helper vector pRK2013 [19]. Briefly, overnight cultures (25 mL of each) of $P$. aeruginosa PAO1 and an E. coli strain containing plasmid pKDpqsR or pRK2013 were centrifuged and the pellets resuspended in phosphate-buffered saline, then mixed in equal proportions and spotted onto an LB agar plate. Following incubation overnight at $37^{\circ} \mathrm{C}$, bacteria were removed from the agar surface and resuspended in $1 \mathrm{~mL}$ of phosphate-buffered saline. Appropriate dilutions were plated onto PIA containing gentamicin at $150 \mu \mathrm{g} \mathrm{mL}^{-1}$. Subsequently, strains that had undergone a second crossover event were selected by plating on LB supplemented with $5 \%$ sucrose and gentamicin. The $p q s R$ gene replacement mutant, designated $\Delta p q s R$, was verified by assessing gentamicin resistance and carbenicillin sensitivity and by PCR analysis.

\subsection{Gene expression assays}

Using the lux-based reporters, gene expression in liquid cultures was measured as counts per second of light production in a Synergy 2 (Biotek, Winooski, Vermont, USA), as previously described by our group [15,20]. Overnight cultures of the reporter strains were diluted to an $A_{600}$ of 0.2 and cultivated for an additional $2 \mathrm{~h}$ before use. The cultures were inoculated into parallel wells of a black 96-well plate with a transparent bottom. A 5- $\mu \mathrm{L}$ volume of the fresh cultures was inoculated into the wells containing a total volume of $95 \mu \mathrm{L}$ medium plus other components, and the $A_{600}$ value in the wells was adjusted to approximately 0.07. A $60-\mu \mathrm{L}$ volume of filter-sterilized mineral oil was added to prevent evaporation during the assay. Promoter activities were measured every $30 \mathrm{~min}$ for $24 \mathrm{~h}$. Bacterial growth was monitored at the same time by measuring the $A_{600}$ in a Syn$\mathrm{ergy}^{2}$. Expression on solid medium was assessed by plating the reporter strains onto soft-top LB agar and imaging using a LAS300 Imaging System (Fuji Corp., Tokyo, Japan).

\subsection{Protein expression and purification}

PmpR protein was expressed as a His-tag fusion using the pProEX-HT protein expression system and purified using the high-affinity nickel-nitrilotriacetic acid resin column (Qiagen, Hilden, Germany) as described previously [15].

\subsection{Electrophoretic Mobility Shift Assay (EMSA)}

The promoter regions of pqsR, exoT, and exs $D$ were PCRamplified according to standard protocols. The template was $P$. aeruginosa genomic DNA. The PCR product and the PmpR protein were incubated for $20 \mathrm{~min}$ at room temperature in binding buffer $\left(40 \mathrm{mmol} \mathrm{L}^{-1}\right.$ Tris- $\mathrm{HCl}$ [pH 7.5], 0.1 mmol L ${ }^{-1}$ EDTA, $70 \mathrm{mmol} \mathrm{L}^{-1} \mathrm{KCl}, 0.1 \mathrm{mmol} \mathrm{L}^{-1}$ dithiothreitol, $4 \mathrm{mmol} \mathrm{L} \mathrm{LgCl}_{2}$ ) and subjected to electrophoresis on a $6 \%$ native polyacrylamide gel. The gel was stained with SYBR green (Invitrogen, Carlsbad, California, USA) and photographed using a LAS-3000 imaging system.

\section{Results}

\subsection{The effect of pmpR on the T3SS}

It has been shown that the quorum-sensing $r h l$ system negatively regulates the T3SS in $P$. aeruginosa [14]. In our previous study, we reported that PmpR regulates the PQS by directly binding to the $p q s R$ promoter region. Considering

Table 2 Primers used in this study

\begin{tabular}{|c|c|c|c|}
\hline Primers & & Sequence $\left(5^{\prime} \rightarrow 3^{\prime}\right)$ & Restriction site (underlined in sequence) \\
\hline \multirow[t]{2}{*}{$p q s R$ upstream } & L1 & GGCGAATTCATCGAGTCCTCGACGCTT & EcoR I \\
\hline & H1 & ATACCCGGGTCGATGGTGATGGCGATA & Sma I \\
\hline \multirow[t]{2}{*}{$p q s R$ downstream } & $\mathrm{L} 2$ & ATACCCGGGCCCGCTTCAACCAGTGCG & Sma I \\
\hline & $\mathrm{H} 2$ & GGCAAGCTTCTACTCTGGTGCGGCGCG & Hind III \\
\hline \multirow[t]{2}{*}{ exs $D$ promoter } & $\mathrm{S} 1$ & CGTCTCGAGCGACATGAGCATCGT & Xho I \\
\hline & $\mathrm{S} 2$ & ATAGGATCCAGCCCACCACGGATACCC & BamHI \\
\hline exoT promoter & $\mathrm{S} 4$ & GA $\underline{\text { GATCCAGTTGTTGCGCCTCCCGG }}$ & BamH I \\
\hline lacZ & lacZf & AGATCGCACTCCAGCCAG & None \\
\hline
\end{tabular}


that the $p m p R$ gene negatively controls $p q s R$ and $r h l R$ expression [16], we speculated that PmpR also controls the T3SS. To test this possibility, we firstly constructed promoter-lux fusions of the T3SS genes exsD, exoS, exoY and exoT. These constructs were integrated into wild-type PAO1 and a $p m p R$-deletion mutant, respectively, and their expression was compared. As shown in Figure 1, the expression of exoS, exoY, exoT and exs $D$ was dramatically increased in the $p m p R$ mutant compared with that in the wild type, while the bacterial growth remained the same (data not shown). The expression of these genes was increased approximately 5 -fold in the $p m p R$ mutant during the stationary phase of growth. This suggests that $p m p R$ negatively regulates expression of the T3SS.

\subsection{PmpR affects the T3SS indirectly}

To test whether the regulation of PmpR on the T3SS is direct, we performed EMSAs using purified PmpR and DNA fragments containing the promoter regions of the T3SS genes. As shown in Figure 2, purified PmpR protein was able to bind to the $p q s R$ promoter region, but could not bind to the promoter regions of exoT or exsD (Figure 2(b)). Similarly, PmpR was not able to bind to either the exoS or exoY promoter region (data not shown). These results indicate that, unlike its effect on $p q s R$ expression, PmpR affects ex- pression of the T3SS indirectly.

\subsection{Regulation of the T3SS by the PQS}

It has been shown that the $r h l$ system negatively regulates the T3SS [14]. In addition, the $r h l$ system negatively regulates the PQS, but the PQS positively regulates the $r h l$ system. To examine the relationships between QS and T3SS, we tested whether the PQS affects the T3SS. The promoterlux fusion constructs of exoS, exoY, exoT, and exsD were integrated into the chromosomes of wild-type PAO1 and the pqs $R$ mutant, respectively, and the expression of these genes was compared. As shown in Figure 3, the expression of ex$o S$ and $e x o T$ was strongly increased in the pqsR mutant, but the expression of exoY and exs $D$ remained the same, indicating that the effect of PqsR on the T3SS is complex and gene-dependent.

\section{Discussion}

Prompted by our previous observations, we investigated the effect of PmpR on the T3SS. We constructed pqsR- and $p m p R$-deletion mutants, and integrated into them promoterreporter fusions of the T3SS genes. We showed that the expression of the T3SS genes exoS, exoY, exoT and exsD
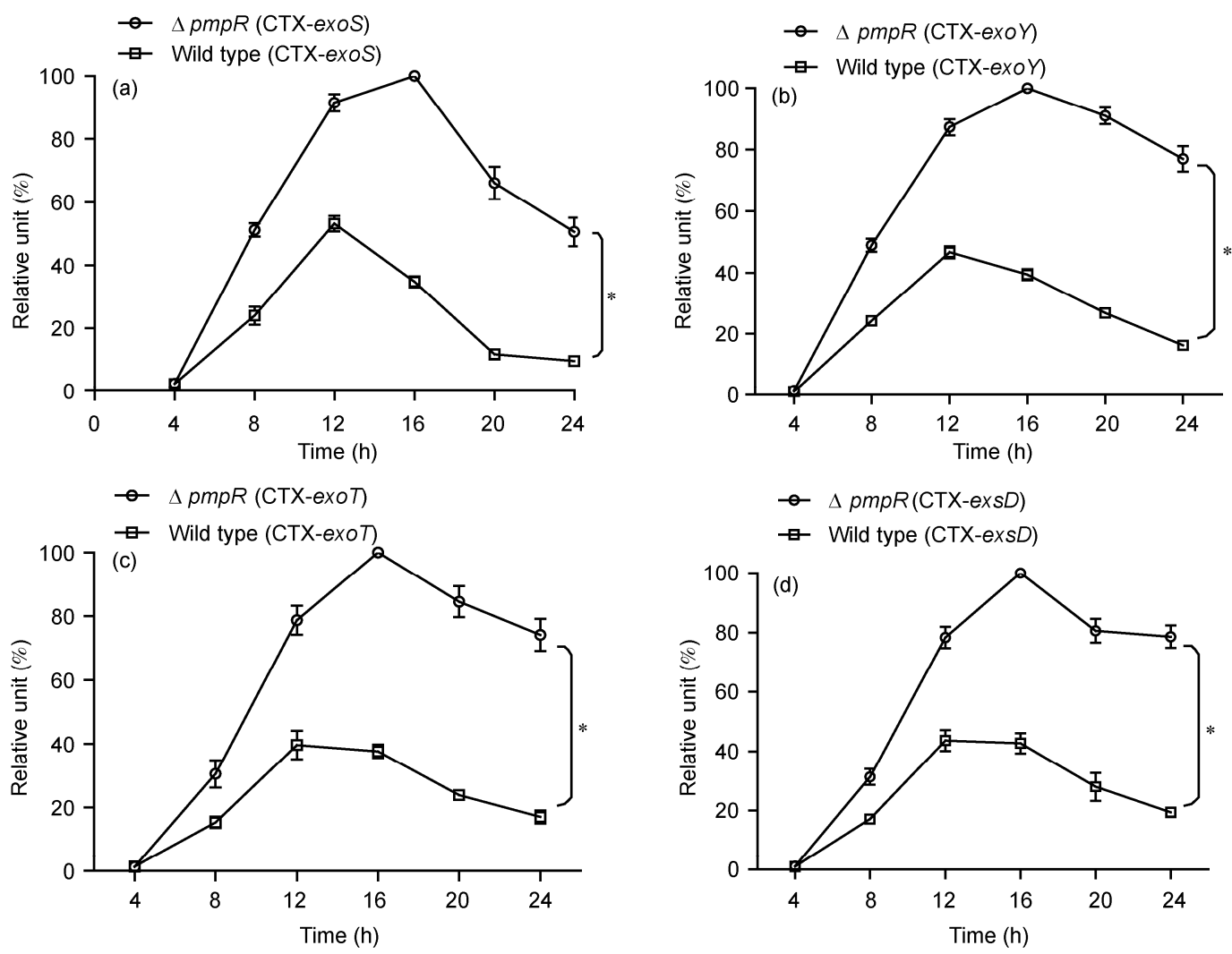

Figure 1 Expression of the T3SS genes (exoS, exoY, exoT, and exs $D)$ in the wild-type PAO1 and the pmpR-deletion mutant. The assays were performed three times and the data are expressed as mean $\pm \mathrm{SD}$. $* P<0.005$, significant difference between the wild type and the mutant (log-rank test). 

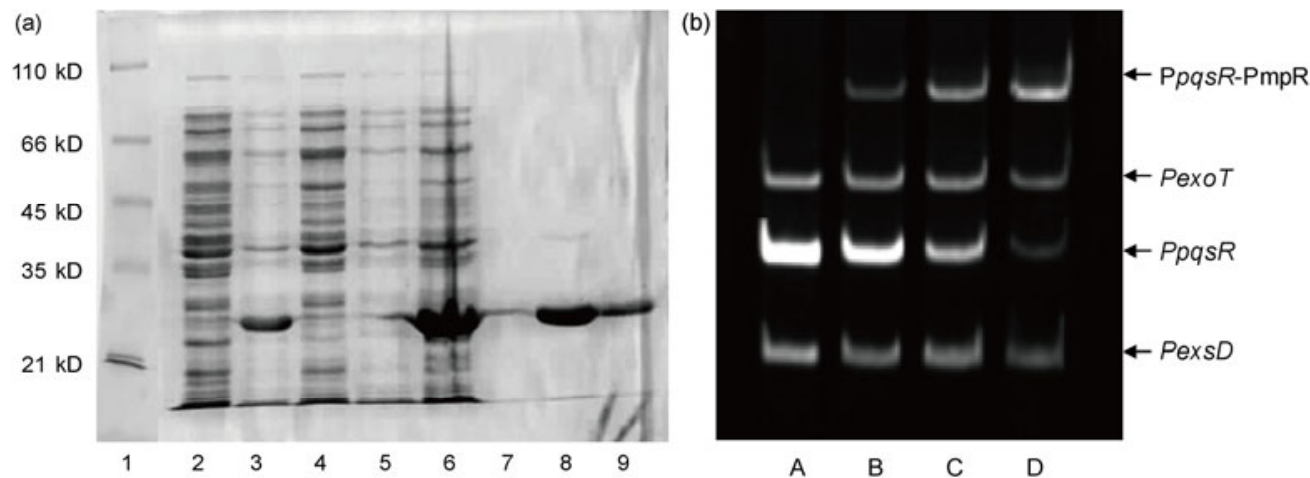

Figure 2 Electrophoretic mobility shift assays (EMSAs). (a) Purification of the PmpR protein. 1, protein marker; 2, cell sample before induction; 3, cell lysate after induction; 4, flow-through solutions from His-column; 5, cell lysate without induction; 6, cell sample after induction; 7-9, purified PmpR protein. (b) EMSA testing of the binding of PmpR to the promoter regions of the T3SS genes. PCR products containing the pqsR, exoT, and exs $D$ promoter regions (PpqsR, PexoT, and PexsD, respectively) were added to the reaction mixtures at approximately 50,30, and $30 \mathrm{ng}$ respectively. The PmpR protein was added to the reaction mixtures in lanes B-D at 320,640 , and $960 \mathrm{ng}$, respectively. No protein was added to the mixture in lane A. DNA containing the $p q s R$ promoter region was bound specifically by PmpR (PpqsR-PmpR). No PmpR binding to the exoT or exsD promoter regions was observed.


Figure 3 Expression of the T3SS genes (exoS, exoY, exoT, and exsD) in wild type and pqsR mutant. These assays were performed three times and the data are expressed as mean $\pm \mathrm{SD}$. $* P<0.005$, significant difference for the expression of exoS and exoT between the wild type and the mutant (log-rank test).

increased dramatically in the $p m p R$ mutant, indicating that PmpR negatively regulates the T3SS. Previously, we demonstrated that PmpR regulates the PQS by directly binding to the $p q s R$ promoter region. Using EMSAs, we showed that PmpR influences the T3SS indirectly.

It is unlikely that PmpR affects the T3SS via the PQS pathway. Although the expression of T3SS genes such as exoS and exoT was strongly increased in the pqsR mutant, the expression of exoY and exsD, however, did not change, indicating that the regulation by PqsR of the T3SS is not the same as that of PmpR, which affected all these genes similarly. In addition, because PmpR negatively regulates both the PQS and the T3SS, and the PQS negatively affects the T3SS, it is not possible for PmpR to affect the T3SS via the PQS system. We speculate that there are other pathways through which PmpR exerts its effect on the T3SS. One 


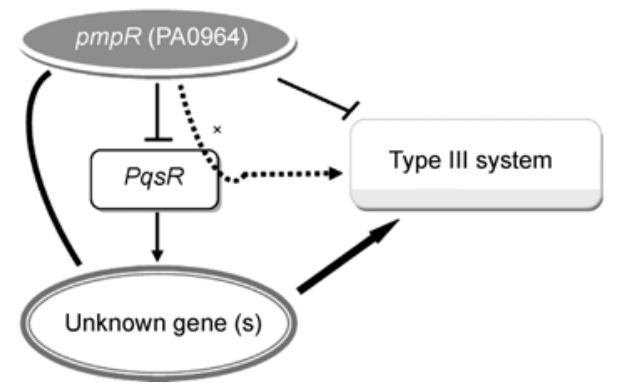

Figure 4 Model of the proposed effect of PmpR on the T3SS. PmpR regulates the T3SS (dashed line) via two possible pathways that are independent of the PQS: 1, PmpR directly regulates the PQS, and the PQS regulates the T3SS through unknown genes (solid black lines); 2, PmpR affects the T3SS via other unknown genes (gray lines). An arrowhead at the end of the line represents positive regulation and a bar at the end of the line represents negative regulation.

possibility is that PmpR first regulates the PQS, and the PQS then regulates the T3SS through some unknown genes (Figure 4). Another possibility is that PmpR influences the T3SS via an unknown pathway that is independent of the PQS. To investigate the regulation pathways, we have constructed a transposon mutagenesis library of $P$. aeruginosa, and are analyzing mutations that alter the effect of PmpR on the expression of T3SS genes. Several interesting genes have been identified. Among them, the expression level of exoS in a pmpR/PA2583 double mutant was the same as in the wild type, and PA2583 directly binds to the exoS promoter region, as shown by EMSA (unpublished data). Therefore, PA2583 is likely to regulate the T3SS positively and represents a candidate protein through which PmpR affects the T3SS. Clearly, the regulatory pathways by which PmpR affects the T3SS are complex. The results reported in this study provide a cue for further investigations.

This work was supported by the National Natural Science Foundation of China (30870097 and 31000049), Shaanxi Province (2010JQ3008 and 2010JK851) and the Northwest University (Doctorate Dissertation of Excellence Fund (08YYB05 and 09NW17)).

1 Pearson J P, Passador L, Iglewski B H, et al. A second $\mathrm{N}$-acylhomoserine lactone signal produced by Pseudomonas aeruginosa. Proc Natl Acad Sci USA, 1995, 92: 1490-1494

2 Quinones B, Dulla G, Lindow S E. Quorum sensing regulates exopolysaccharide production, motility, and virulence in Pseudomonas syringae. Mol Plant Microbe Interact, 2005, 18: 682-693

3 Latifi A, Foglino M, Tanaka K, et al. A hierarchical quorum-sensing cascade in Pseudomonas aeruginosa links the transcriptional activators LasR and RhIR (VsmR) to expression of the stationaryphase sigma factor RpoS. Mol Microbiol, 1996, 21: 1137-1146

4 Schuster M, Lostroh C P, Ogi T, et al. Identification, timing, and signal specificity of Pseudomonas aeruginosa quorum-controlled genes: A transcriptome analysis. J Bacteriol, 2003, 185: 2066-2079

5 Wagner V E, Bushnell D, Passador L, et al. Microarray analysis of Pseudomonas aeruginosa quorum-sensing regulons: Effects of growth phase and environment. J Bacteriol, 2003, 185: 2080-2095

6 Pesci E C, Milbank J B J, Pearson J P, et al. Quinolone signaling in the cell-to-cell communication system of Pseudomonas aeruginosa. Proc Natl Acad Sci USA, 1999, 96: 11229-11234

7 Diggle $\mathrm{S}$ P, Cornelis $\mathrm{P}$, Williams $\mathrm{P}$, et al. 4-quinolone signalling in Pseudomonas aeruginosa: Old molecules, new perspectives. Int $\mathrm{J}$ Med Microbiol, 2006, 296: 83-91

8 Déziel E, Lépine F, Milot S, et al. Analysis of Pseudomonas aeruginosa 4-hydroxy-2-alkylquinolines (HAQs) reveals a role for 4-hydroxy-2-heptylquinoline in cell-to-cell communication. Proc Natl Acad Sci USA, 2004, 101: 1339-1344

9 Gallagher LA, McKnight S L, Kuznetsova M S, et al. Functions required for extracellular quinolone signaling by Pseudomonas aeruginosa. J Bacteriol, 2002, 184: 6472-6480

10 Frank D W. The exoenzyme S regulon of Pseudomonas aeruginosa. Mol Microbiol, 1997, 26: 621-629

11 Dasgupta N, Lykken G L, Wolfgang M C, et al. A novel anti-antiactivator mechanism regulates expression of the Pseudomonas aeruginosa type III secretion system. Mol Microbiol, 2004, 53: 297-308

12 Kim J, Ahn K, Min S, et al. Factors triggering type III secretion in Pseudomonas aeruginosa. Microbiology, 2005, 151: 3575-3587

13 McCaw M L, Lykken G L, Singh P K, et al. ExsD is a negative regulator of the Pseudomonas aeruginosa type III secretion regulon. Mol Microbiol, 2002, 46: 1123-1133

14 Bleves S, Soscia C, Nogueira-Orlandi P, et al. Quorum sensing negatively controls type III secretion regulon expression in Pseudomonas aeruginosa PAO1. J Bacteriol, 2005, 187: 3898-3902

15 Liang H, Li L, Dong Z, et al. The YebC family protein PA0964 negatively regulates the Pseudomonas aeruginosa quinolone signal system and pyocyanin production. J Bacteriol, 2008, 190: 6217-6227

16 Simon R, Priefer U, Pühler A. A broad host range mobilization system for in vivo genetic engineering: Transposon mutagenesis in gram negative bacteria. Bio/Technology, 1983, 1: 784-791

17 Hoang T T, Karkhoff-Schweizer R R, Kutchma A J, et al. A broadhost-range Flp-FRT recombination system for site-specific excision of chromosomally-located DNA sequences: Application for isolation of unmarked Pseudomonas aeruginosa mutants. Gene, 1998, 212: 77-86

18 Schweizer H P. Two plasmids, X1918 and Z1918, for easy recovery of the $x y l E$ and $l a c Z$ reporter genes. Gene, 1993, 134: 89-91

19 Ditta G, Stanfield S, Corbin D, et al. Broad host range DNA cloning system for gram-negative bacteria: Construction of a gene bank of Rhizobium meliloti. Proc Natl Acad Sci USA, 1980, 77: 7347-7351

20 Duan K, Dammel C, Stein J, et al. Modulation of Pseudomonas aeruginosa gene expression by host microflora through interspecies communication. Mol Microbiol, 2003, 50: 1477-1491

21 Stover C K, Pham X Q, Erwin A L, et al. Complete genome sequence of Pseudomonas aeruginosa PA01, an opportunistic pathogen. Nature, 2000, 406: 959-964

Open Access This article is distributed under the terms of the Creative Commons Attribution License which permits any use, distribution, and reproduction in any medium, provided the original author(s) and source are credited. 